\title{
Marginal Metric Utility for Autonomic Cloud Application Management
}

\author{
Marta Różańska \\ University of Oslo \\ Oslo, Norway \\ martaroz@ifi.uio.no
}

\author{
Geir Horn \\ University of Oslo \\ Oslo, Norway \\ Geir.Horn@mn.uio.no
}

\begin{abstract}
Managing Cloud applications with variable resource requirements over time is an insipid task that could benefit from autonomic application management. The management platform will then need to know what the application owner considers a good deployment for the current execution context, which is normally captured by a utility function. However, it is often difficult to define such a function directly by first principles in a way that would perfectly capture the application owner's preferences. This paper proposes a methodology for defining the utility function only from the monitoring measurements taken to assess the state and context of the running application.
\end{abstract}

\section{CCS CONCEPTS}

- Software and its engineering $\rightarrow$ Cloud computing; • Computing methodologies $\rightarrow$ Modeling methodologies; • Applied computing $\rightarrow$ Multi-criterion optimization and decision-making.

\section{KEYWORDS}

cloud computing, utility function modelling, multi-criteria decision making

\section{ACM Reference Format:}

Marta Różańska and Geir Horn. 2021. Marginal Metric Utility for Autonomic Cloud Application Management. In 2021 IEEE/ACM 14th International Conference on Utility and Cloud Computing (UCC '21) Companion (UCC '21 Companion), December 6-9, 2021, Leicester, United Kingdom. ACM, New York, NY, USA, 8 pages. https://doi.org/10.1145/3492323.3495587

\section{INTRODUCTION}

Utility computing in general, and Cloud computing in particular, are based on the fundamental idea that computing resources can be acquired when needed and released when they are no longer of use. Only applications whose resource needs are varying over time can truly benefit from this concept. The implication is that someone must manage these applications and monitor their resource pool to ensure that the necessary resources are provided or released timely. Furthermore, resource scaling and elasticity is beneficial mostly for applications running for some time. However, the cost of continuously managing manually the application may prevent the

ACM acknowledges that this contribution was authored or co-authored by an employee, contractor or affiliate of a national government. As such, the Government retains a nonexclusive, royalty-free right to publish or reproduce this article, or to allow others to do so, for Government purposes only.

UCC '21 Companion, December 6-9, 2021, Leicester, United Kingdom

(C) 2021 Association for Computing Machinery.

ACM ISBN 978-1-4503-9163-4/21/12 . \$ \$15.00

https://doi.org/10.1145/3492323.3495587 organization owning the application from harvesting the benefits offered by the infrastructure elasticity.

The only solution is to let the application be managed by an autonomic computing deployment platform building on the ideas from the vision of 'autonomic computing' [12]. The core building block in this architecture is the Monitor, Analyse, Plan, Execute - with Knowledge (MAPE-K) [9] feedback loop. This concept has been used to build management frameworks for mobile applications [14], for ubiquitous computing systems [19], and recently for autonomic management of applications deployed simultaneously across multiple Cloud providers [8].

Monitoring the application's execution and context is the first and essential part of realizing the MAPE-K feedback loop. The monitored values will change over the deployed application's lifetime even if the application's deployment configuration is left unchanged. Users may come and go, data processed by the algorithms may require more or less computational time, the Cloud provider may multiplex more or less Virtual Machines (VMs) on the same physical server, and so forth. We will later present an example application where documents are kept encrypted in a database and decrypted by a server on users' demand. It is clear that the extreme case of deploying one decryption server per user will give each user the highest satisfaction level possible, but also the largest deployment cost. On the other hand, multiplexing too many users on each decryption server may give too long response times and eventually turn paying users away and thereby reduce the owning organisation's income from operating the application. Hence, the number of decryption servers impacts both the cost and the users' satisfaction.

The main idea behind this paper is that the DevOps engineers and application owners have a better understanding of how the application's utility will vary with the changes in each of the monitored values. Returning to our example: Say that one measures the response time for a user from the document request is made until the decoded document has been received by the user. The best utility will be if this response time, normalized to the size of the requested document, stays around some nominal value. If response time drops too low it means that too many servers are provided and the deployment is too costly, and if it grows too high application users may become unsatisfied.

This paper proposes a novel methodology for modelling utility functions useful in autonomic computing systems based on deciding the individual utilities for each monitored value. These utility functions are then combined to decide on the overall change in the application's utility that may be used by the autonomic application management platform to decide on a better deployment configuration for the application. 
Our proposed methodology will be presented in Section 4 after first discussing utility functions in autonomic managements in Section 2 and the marginal metric utility modelling approach in Section 3. We will propose some illustrative functions in Section 4.2 that are taken from the class of exponential functions as this class has previously been shown to have useful properties for utility function modelling [16]. We will use the encrypted document example application in Section 5 where the utility function will initially be modelled by first principles, and then by our proposed method. The two utility representations are evaluated against queueing theory results in Section 6 with an assessment of the approach in Section 7.

\section{UTILITY FUNCTION IN AUTONOMIC APPLICATION MANAGEMENT}

An autonomic application management platform will, by definition, make decisions on behalf of the organization owning the deployed application. These decisions are valid only if they concur with the decisions that otherwise would have been made by the human DevOps engineers in these organizations. Rational choice theory understands human economic decisions and social behaviour as maximizing the decison maker's subjective utility [10]. The utility is almost certainly multidimensional combining objectives measured on different scales. Overall, the multiple objectives can be conflicting and the utility function balances the different utility dimensions. Two utility dimensions are typically present in all Cloud deployments: the deployment cost measured in some monetary unit, and the application performance measured in a different unit.

The challenge for all autonomic computing platforms is therefore to capture the application owners' utility and maximise this utility given the current use of the application and its execution context. Kephart and Das advocated that the best way to represent utility in autonomic systems was as a utility function [13]. This approach has been taken by all the above mentioned frameworks and platforms. However, the main difficulty in modelling the utility function is capturing the interplay of the application's deployment configuration and its execution context. Returning to our example: the number of decryption servers impacts both the cost and the users' satisfaction, and ultimately the benefits of operating this application in ways that may be challenging to model and represent mathematically in a single function. Experiments conducted with experienced DevOps engineers have shown that formulating explicitly the utility function for an application is hard and discouraging [11].

Despite the fact that utility functions are used in both self-adaptive systems and Cloud management platforms, the actual modelling of the utility function has not received any attention as far as we are aware. The starting point for utility modelling is the concept of the utility attributes used by the decision maker to decide on the utility, i.e. the utility attributes correspond to the utility dimensions. Keenley showed that if the attributes are independent, then the utility function is either additive or multiplicative in individual marginal univariate utility functions over the individual utility attributes [17].

There is an approach provided that there is systematic variation in the utility attribute values implying that more of every utility attribute is better than less. If one additionally has that the utility will be zero whenever any single attribute attains its minimum value, then the utility function will belong to the class of attribute dominance utility functions [2]. This class has similar mathematical properties as multivariate joint cumulative probability distributions, and the joint utility function can be derived from the univariate 'marginal' utility functions for each of the utility dimensions. Abbas and Howard suggested using copula theory [18] developed for joint probability distributions to model attribute dominant utility, although this would natively result in a utility function where all mixed partial derivatives with respect to the attribute dimensions would be positive, which is called an $n$-increasing utility [2]. This consequence can be undesireable for many utility functions. Abbas subsequently proposed specialized utility copulas relaxing the requirement of zero utility at the lower limit of any single attribute and the $n$-increasing property [1], and defined the Archimedean utility copulas inspired by the similar copula concept from statistics [6]. Abbas and Sun developed a methodology for constructing an Archimedean utility copula from a set of preferences given by the decision maker [3], and recently they analysed a form of the Archimedean utility copula with polynomial generating functions [4].

It should be noted that the marginal utility functions used for the utility copula are functions of the decision attributes. In other words, the utility copula theory provides an excellent framework for combining the utility dimensions for autonomic Cloud application management. However, the application owner must still define the link between the marginal utility and the possible actions and system state represented respectively by the configuration and the application execution context. Our proposed approach closes this gap with a methodology for directly modelling each marginal utility dimension based on the decision maker's assessment on the impact of the application execution context only.

\section{MARGINAL METRIC UTILITY MODELLING}

A Cloud application is understood as a set of connected components, $\mathbb{C}$. Each component, $C \in \mathbb{C}$, has a set of requirement attributes, $\mathbb{A}_{C}$. These typically specifies the resource necessary for the component to perform as expected. Thus, a requirement attribute can be the number of cores useful for the component ranging from a minimum number to a maximum number, or it can be the amount of memory, or the Cloud providers that can be used, or the geographic location of the hosting data centre, or the number of copies or instances of the component the application can successfully exploit. The requirment attributes can be continuous or discrete. Each value of a requirement attribute value, $a_{C, i}$, is taken from the attribute's domain, $a_{C, i} \in \mathbb{A}_{C, i}$, for all the requirement attributes $i=1, \ldots\left|\mathbb{A}_{C}\right|$.

A configuration of a component is an assignment of values to all its requirement attributes. The different ways a component $C$ can be configured is its variability space, which is the Cartesian product of its attribute domains, $\mathbb{V}_{C}=\mathbb{A}_{C, 1} \times \cdots \times \mathbb{A}_{C,\left|\mathbb{A}_{C}\right|}$. The configuration of the Cloud application is a flattened vector, $\boldsymbol{c}$, of the configurations of all the components of the application. The variability space of the application is therefore the Cartesian product of the variability spaces for its components, $\mathbb{V}=\mathbb{V}_{1} \times \cdots \times \mathbb{V}_{|\mathbb{C}|}$.

The utility function for the deployment is a mapping from the application configuration to a "goodness factor" in the unit interval, $U: c \in \mathbb{V} \mapsto[0,1]$. However, a configuration giving a good utility at the current time, $t_{k}$, may well not give a good utility if the 
application's execution context changes. This could be application users arriving or departing, or changes in the data processed by the application, or anything else that will impact the application's performance or behaviour. The application must be monitored to detect these changes as described in the Introduction. The utility is therefore conditioned on the monitored values jointly referred to as the application's execution context, $\boldsymbol{\theta}\left(t_{k}\right)$. Furthermore, there can be some fixed parameters needed to decide the shape of the utility function and these are denoted $\phi$. The utility function is therefore a function of the configuration vector given the measurement vector and the parameter vector: $U\left(\boldsymbol{c}\left(t_{k}\right) \mid \boldsymbol{\theta}\left(t_{k}\right), \boldsymbol{\phi}\right)$.

It should be noted that the configuration has been given a time index because it is valid only for the current execution context, $\boldsymbol{\theta}\left(t_{k}\right)$. The current context will change whenever there is an update available for any of the measured values. These context changes are generally irregular events, for instance caused by the arrival or departure of an application user. The ultimate goal of the DevOps engineer or the autonomic platform managing the application will be to find the best configuration, $c^{*}\left(t_{k}\right)$ for the current context: $\boldsymbol{c}^{*}\left(t_{k}\right)=\operatorname{argmax}_{\boldsymbol{c}\left(t_{k}\right) \in \mathbb{V}} U\left(\boldsymbol{c}\left(t_{k}\right) \mid \boldsymbol{\theta}\left(t_{k}\right), \boldsymbol{\phi}\right)$.

It may take time to solve this optimization problem since the search space, $\mathbb{V}$, can be large. Furthermore, if there are many measurements taken from the application one may expect that the interval between two measurement events is short and consequently that the execution context will change frequently. In practice, one will therefore keep one configuration for multiple measurement events and observe the degradation in the utility value to decide when it is necessary to reconfigure the application.

When managing the Cloud application manually, the DevOps engineer will need to decide on an initial deployment configuration for the application and then subsequently a new configuration at some time $t_{k}$ when the current configuration is considered no longer optimal. This means that the DevOps engineer will decide a configuration $c^{*}\left(t_{k}\right)$ that is deemed optimal for the current application execution context $\boldsymbol{\theta}\left(t_{k}\right)$ at this point in time. There is no need to change this perceived optimal configuration unless the changes in the utility value at some later time $t_{k+h}$ indicates that reconfiguration actions must be taken. The change in the utility value over the time interval $\left[t_{k}, t_{k+h}\right]$ is therefore solely driven by the changes in the application's execution context. The question we try to answer in this paper is: How can the DevOps engineer estimate the change in utility for the configuration $c^{*}\left(t_{k}\right)$ based on the observed current execution context $\boldsymbol{\theta}\left(t_{t+h}\right)$ ?

Let the DevOps engineer define utility functions for each of the measured metric values, $u_{i}\left(\theta_{i}\right)$. These can then be taken as the marginal utilities. The utility function $U\left(\boldsymbol{c}\left(t_{k}\right) \mid \boldsymbol{\theta}\left(t_{k+h}\right), \boldsymbol{\phi}\right)$ is essentially a multivariate function of all configuration variables $c\left(t_{k}\right)$ and the given measurements $\boldsymbol{\theta}\left(t_{k}\right)$. The linearized utility around an optimal configuration $c^{*}\left(t_{k}\right)=\left[c_{1}^{*}\left(t_{k}\right), c_{2}^{*}\left(t_{k}\right), \ldots, c_{n}^{*}\left(t_{k}\right)\right]^{T}$ and the application execution context $\boldsymbol{\theta}\left(t_{k}\right)=\left[\theta_{1}\left(t_{k}\right), \theta_{2}\left(t_{k}\right), \ldots, \theta_{m}\left(t_{k}\right)\right]^{T}$, for which $c^{*}\left(t_{k}\right)$ is deemed to be the optimal configuration, can be written as

$$
\begin{array}{r}
U\left(c^{*}\left(t_{k}\right) \mid \boldsymbol{\theta}\left(t_{k+h}\right), \boldsymbol{\phi}\right)=U\left(\boldsymbol{c}^{*}\left(t_{k}\right) \mid \boldsymbol{\theta}\left(t_{k}\right), \boldsymbol{\phi}\right) \\
+\left.\sum_{i=1}^{m} \frac{\partial u_{i}}{\partial \theta_{i}}\right|_{\left(\boldsymbol{c}^{*}\left(t_{k}\right), \boldsymbol{\theta}\left(t_{k}\right)\right)}\left[\theta_{i}\left(t_{k+h}\right)-\theta_{i}\left(t_{k}\right)\right]
\end{array}
$$

\section{MARGINAL METRIC TEMPLATE UTILITY}

\subsection{Method for modelling utility function}

The proposed method for retrieving DevOps engineer's preferences in accordance with the application's optimization goals is presented schematically in Algorithm 1. It should be noted that it is not a pseudocode but a schema of steps that are needed and can be implemented in various ways. First, the DevOps engineer is asked to define the vector of metrics, $\boldsymbol{\theta}(t)$. Then a 'template' marginal metric utility function for each metric $\theta_{i}(t) \in \boldsymbol{\theta}(t)$ must be chosen. We describe the details of the proposed template functions, $f=\left[f_{1}, f_{2}, \ldots, f_{5}\right]$ in Section 4.2 where we discuss their desirable properties. However, any new template function can be added to $f$ without any changes in Algorithm 1 provided this new function is differentiable, as it was necessary for the linearized approach of Section 3.

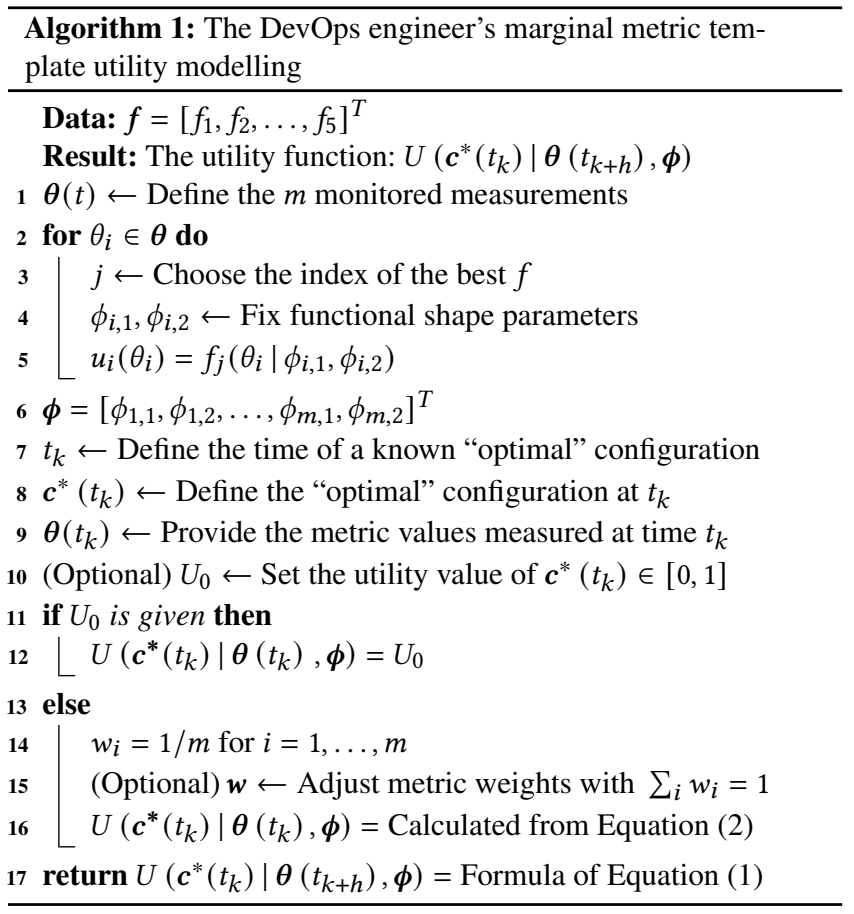

It should be noted that fixing the utility value for $c^{*}\left(t_{k}\right)$ at one of the extremes will have a bearing on the marginal metric 'template' utility function forms and possibly the time of validity of the linearization because the last term of (1) must be strictly positive if $U\left(c^{*}\left(t_{k}\right) \mid \boldsymbol{\theta}\left(t_{k}\right), \boldsymbol{\phi}\right)=0$ or it must be strictly negative if $U\left(c^{*}\left(t_{k}\right) \mid \boldsymbol{\theta}\left(t_{k}\right), \boldsymbol{\phi}\right)=1$. If the DevOps engineer can assign a weight of importance, $w_{i} \in[0,1]$, to the utility of measurement $\theta_{i}(t)$ such that the weights sum to unity, $\sum_{i} w_{i}=1$, it is recommended to take the utility value of the optimal configuration $c\left(t_{k}\right)$ as the affine combination of the utilities of the metric values,

$$
U\left(c^{*}\left(t_{k}\right) \mid \boldsymbol{\theta}\left(t_{k}\right), \boldsymbol{\phi}\right)=\sum_{i=1}^{m} w_{i} \cdot u_{i}\left(\theta_{i}\left(t_{k}\right)\right)
$$

\subsection{Template function shapes}

We propose some illustrative functions that represent typical deployment goals regarding a monitored value like "keep it close to 


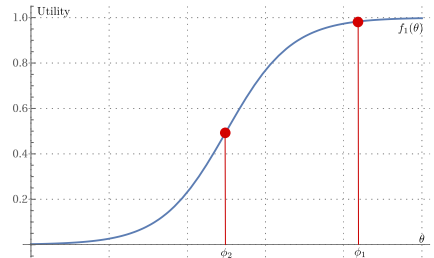

(a) Template S-shaped sigmoid function

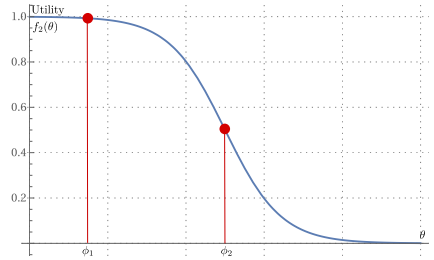

(b) Template for Reversed S-shaped Sigmoid function

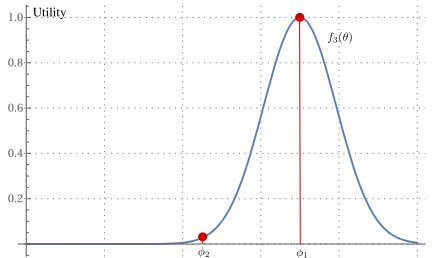

(c) Template Bell shaped function

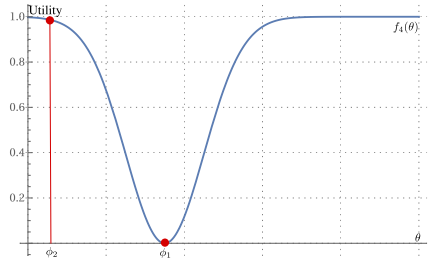

(d) Template U-shaped function

Figure 1: Four of the metric 'template' functions proposed

a reference value" or "as high as possible" or "as low as possible". Furthermore, all our 'templates' are taken from the class of exponential functions as this class has previously been shown to have useful properties for utility function modelling [15]. Furthermore there are templates proposed by Chang [5]. Additionally, we propose to use two functions that we call the U-shaped utility function and Bell shaped utility function to have the possibility to represent the goal "keep the monitored value close to a reference value". The suggested list of 'template' functions is far from exhaustive and only meant as a starting point to illustrate the proposed methodology.

4.2.1 S-shaped sigmoid function. The sigmoid function $f_{1}$ can be seen as a similar function to the standard 'S-shaped' utility function, which is one of of the most popular shapes of utility functions [15], modified to be differentiable. This function has a shape presented in Figure 1a, where $\theta=\theta_{i}\left(t_{k}\right)$ is the chosen metric. The motivation behind choosing this template function may be the goal to achieve the highest possible monitored metric value. When choosing this function, there is a need to indicate two points: $\phi_{1}$ where the utility value is close to 1 and $\phi_{2}<\phi_{1}$ where the value should be 0.5 . For these two points: $\phi_{1}$ and $\phi_{2}$, it is possible to solve the shape parameters of the general function to calculate the function form (3).

$$
f_{1}\left(\theta \mid \phi_{1}, \phi_{2}\right)=1-\left[1+e^{\left(\left(\theta-\phi_{2}\right) \ln [(1-\epsilon) / \epsilon]\right) /\left(\phi_{1}-\phi_{2}\right)}\right]^{-1}
$$

4.2.2 Reversed S-shaped sigmoid function. As can be seen of Figure $1 \mathrm{~b}$, it is a very similar function to $\mathrm{S}$-shaped sigmoid function. The only difference is that for bigger values of the metric value, the function value decreases. This template can be used to express the preference that the monitored value should be as low as possible. When choosing this function, there is a need to indicate two points: $\phi_{1}$ where the utility value is close to 1 and $\phi_{2}>\phi_{1}$ where the value should be 0.5 . For these two points, it is possible to solve the shape parameters of the general sigmoid function to calculate the function form, which results as (4).

$$
f_{2}\left(\theta \mid \phi_{1}, \phi_{2}\right)=\left[1+e^{\left(\left(\theta-\phi_{2}\right) \ln [\epsilon /(1-\epsilon)]\right) /\left(\phi_{1}-\phi_{2}\right)}\right]^{-1}
$$

4.2.3 Bell shaped function. This function is our new proposal for a utility function template. The intuition behind it is simple: there is a nominal metric value that DevOps engineer expects to be achieved, and the more the measured values are deviating from the expected value, the lower the utility is for the application owner. This function $f_{3}(\theta)$ has a shape which can be seen in Figure 1c, where $\theta$ is the metric value. Having this function chosen as a template, there is a need to indicate two points: $\phi_{1}$, which is the nominal metric value, where the utility value is equal to one, and $\phi_{2}<\phi_{1}$ where the value is close to zero. For these two points: $\phi_{1}$ and $\phi_{2}$, it is possible to solve for the shape parameters of the general function to calculate the function form, which results as (5).

$$
f_{3}\left(\theta \mid \phi_{1}, \phi_{2}\right)=e^{\ln \epsilon\left(\theta-\phi_{1}\right)^{2} /\left(\phi_{2}-\phi_{1}\right)^{2}}
$$

4.2.4U-shaped function. U-shaped function has a shape presented on Figure 1d. It is a second new proposal for the utility function template. The motivation behind this function is similar to the Bell shaped template. It can be used when user would like to express the preference to avoid one precise value of the measured metric $\theta$. Having this function chosen as a template, there is a need to indicate two points: $\phi_{1}$ where the utility value is equal to zero, and $\phi_{2}<\phi_{1}$ where the value is close to one. The general function has a form (6).

$$
f_{4}\left(\theta \mid \phi_{1}, \phi_{2}\right)=1-e^{-\ln \epsilon\left(\theta-\phi_{1}\right)^{2} /\left(\phi_{2}-\phi_{1}\right)^{2}}
$$

4.2.5 Constant function. The constant function is a very simple function that can be used to indicate that the measured metric $\theta$ is not important for the user in terms of the utility. If the user chooses this function as a template, there is a need to indicate one value: $\phi_{1} \in[0,1]$, which is achieved independently on the $\theta$ value.

$$
f_{5}\left(\theta \mid \phi_{1}\right)=\phi_{1}=0
$$

\section{SECURE STORAGE EXAMPLE}

\subsection{Description}

Our approach can be illustrated with an example based on the software application of MELODIC project use case provider ${ }^{1}$ to store documents in a secure way mentioned in Section 1 that was introduced in Horn and Skrzypek [8] and used by Horn and Rozanska [7]. The software serves the possibility to store encrypted documents in a secure way and its owner has the deployment goal to provide the best possible performance to the users. The performance is measured in terms of the average response time to the users, but the deployment should be done at the minimal price per served request. There are two main decision variables: $c_{1}$, which is the number of application server instances, and $c_{2}$, which is the number of cores available at each instance. It is assumed that one document decoding thread runs on one core only. Hence, the number of 'servers' seen from the application owner's perspective is equal to the number of

\footnotetext{
${ }^{1}$ https://h2020.melodic.cloud/secure-documents-success-story/
} 
cores. Furthermore, let us assume the execution context of of the application is defined by the following metrics:

(1) $\theta_{1}(t)$, which is the average response time to the user,

(2) $\theta_{2}(t)$, which is the number of requests to store or get a document that are coming to the application per second, and

(3) $\theta_{3}(t)$, which is the fraction of requests per second that are unacceptably delayed from the users' perspective.

The problem has two utility dimensions: One is the performance where the response time experienced for each user should be kept at an acceptable level; and the other is the desire to use as few servers as possible to limit the deployment cost.

The utility functions will be modelled using two approaches: the direct utility modelling in Section 5.2 and the marginal metric utility function modelling approach proposed in this paper in Section 5.3. This example creates the baseline for the evaluation of the method in Section 6.

\subsection{Direct utility function modelling}

In this section, we propose two directly modelled utility functions, one for each utility dimension. We combine both utility dimensions functions into an overall utility in an affine way.

5.2.1 Performance utility. From the performance point of view, it would obviously be best if each user had a dedicated virtual machine, as this would minimize the response time. However, this would inflate the cost of the deployment. Therefore, it is more appropriate to define the expected response time $\bar{T}$ and the maximum acceptable response time $T^{+}$as a threshold that should not be exceeded. This utility function has been formulated by Horn and Rozanska [7] and we simply use the same formula as $v_{\text {time }}\left(c^{*}\left(t_{k}\right) \mid \boldsymbol{\theta}\left(t_{k+h}\right), \boldsymbol{\phi}\right)$.

5.2.2 Cost utility. The utility is best captured using the cost per served user as a parameter. We note that the more users can be served on the VM, the relative cost is lower. Let $P^{+}$and $P^{-}$be the maximum and the minimum prices for the single available VMs. Let $c_{1}{ }^{+}$and $c_{1}{ }^{-}$be the maximum and the minimum number of decoding servers which is limited by the application owner, and $P\left(c^{*}\left(t_{k}\right)\right)$ be the price of deployment represented by $c^{*}\left(t_{k}\right)$. The cost utility can be expressed as:

$$
\begin{aligned}
& v_{\text {price }}\left(\boldsymbol{c}^{*}\left(t_{k}\right) \mid \boldsymbol{\theta}\left(t_{k+h}\right), \boldsymbol{\phi}\right)= \\
& \frac{\theta_{2}\left(t_{k+h}\right) \cdot P^{+} \cdot c_{1}^{+}-P\left(\boldsymbol{c}^{*}\left(t_{k}\right)\right) /\left(1-\theta_{3}\left(t_{k+h}\right)\right)}{\theta_{2}\left(t_{k+h}\right) \cdot P^{+} \cdot c_{1}{ }^{+}-P^{-} \cdot c_{1}}
\end{aligned}
$$

which is similar function to the standard normalization function $(x-\min (x)) /(\max (x)-\min (x))$. The numerator is changed to $\max (x)-x$ to reflect the fact that minimum price gives the highest cost utility. The main parameter is the price of the configuration divided by the ratio of served requests, which gives the information about the deployment cost per served request. The ratio of served requests is calculated as $1-\theta_{3}(t)$. The maximum value is calculated as the most expensive deployment, which is the biggest possible number of the most costly VMs divided by the worst possible ratio of served requests, which is only one from all requests received in one second: $\left(P^{+} \cdot c_{1}{ }^{+}\right) /\left[1-\left(\theta_{2}(t)-1\right) / \theta_{2}(t)\right]$. After simplification, the form of the maximum value is $\theta_{2}(t) \cdot P^{+} \cdot c_{1}{ }^{+}$. The minimum price for the deployment is calculated as the minimum number of the least expensive machines divided by the best possible ratio of served requests that is one, $1 \cdot P^{-} \cdot c_{1}{ }^{-}$.

5.2.3 Overall utility. The overall utility contain two dimensions: performance and cost. Considering these two parts of utility function, the overall utility function is modelled as the affine combination of the two dimensions:

$$
\begin{aligned}
v\left(\mathbf{c}^{*}\left(t_{k}\right) \mid \boldsymbol{\theta}\left(t_{k+h}\right), \boldsymbol{\phi}\right) & =\zeta v_{\text {time }}\left(\mathbf{c}^{*}\left(t_{k}\right) \mid \boldsymbol{\theta}\left(t_{k+h}\right), \boldsymbol{\phi}\right) \\
& +(1-\zeta) v_{\text {price }}\left(\mathbf{c}^{*}\left(t_{k}\right) \mid \boldsymbol{\theta}\left(t_{k+h}\right), \boldsymbol{\phi}\right)
\end{aligned}
$$

The weight $\zeta$ can be computed by using the Pareto Front of the bi-objective optimisation problem [7].

\subsection{Marginal metric utility function modelling}

According to the modelling approach proposed in this paper, there is no need for direct modelling of the utility function formula. It is enough to specify functions for each metric for each dimension $\theta_{i}(t) \in \boldsymbol{\theta}(t)$, in this example the performance and cost.

5.3.1 Performance utility modelling. There is a need to choose template functions that can be used as $u_{1}, u_{2}, u_{3}$ for the performance dimension. Performance utility considers the average response time to the user and for this purpose only the average response time metric $\theta_{1}$ is used. The marginal metric utility functions for other metrics should be constant.

A function that evaluates the average response time $\theta_{1}$ should return the highest possible value if the average response time is equal to the best possible value which is $\bar{T}_{S}$, i.e. the average response time of directly served requests. It should have the lowest value when the average response time is significantly greater than the response time $T^{+}$that makes the request considered as delayed but still served. These are two points that the DevOps engineer should define to calculate the shape of the template function $f_{3}$, which is considered the most suitable in this case:

$$
u_{1}\left(\theta_{1}(t) \mid \boldsymbol{\phi}\right)=f_{3}\left(\theta_{1}(t) \mid \phi_{1,1}=\bar{T}_{S}, \phi_{1,2}=T^{+}\right)
$$

The number of requests $\theta_{2}(t)$ and the fraction of delayed requests $\theta_{3}(t)$ are a part the execution context for the user in terms of the performance utility. It is the objective information that should be considered during the optimization process, but generally every number of users gives the same performance utility for the application owner as long as users are properly served. Therefore, the marginal metrics utility functions can be modelled as constant functions $f_{5}$ :

$$
u_{2}\left(\theta_{2}(t) \mid \boldsymbol{\phi}\right)=u_{3}\left(\theta_{3}(t) \mid \boldsymbol{\phi}\right)=f_{5}\left(\theta_{2}(t) \mid \phi_{2,1}=1\right)=1 .
$$

We define the performance utility from (1), using (10):

$$
\begin{aligned}
U_{\text {time }} & \left(\boldsymbol{c}\left(t_{k}\right) \mid \boldsymbol{\theta}\left(t_{k+h}\right), \boldsymbol{\phi}\right) \\
& =u_{1}\left(\theta_{1}\left(t_{k}\right) \mid \boldsymbol{\phi}\right)+\frac{\partial u_{1}}{\partial \theta_{1}}\left(\theta_{1}\left(t_{k}\right)\left[\theta_{1}\left(t_{k+h}\right)-\theta_{1}\left(t_{k}\right)\right]\right.
\end{aligned}
$$

where the first part of the sum is $u_{1}\left(\theta_{1}\left(t_{k}\right)\right)$ derived from (2) with the assumption that weights $w_{2}, w_{3}$ are zero.

5.3.2 Cost utility modelling. There is a need to choose template functions that can be used as $u_{1}, u_{2}, u_{3}$ for the cost dimension. The cost utility should express the desire of the application's owner to minimize the cost per the served user. Therefore, the first metric that gives the information about the average response time does not need 
to be considered. In this cost utility function, only two metrics are involved: $\theta_{2}(t)$, which is the number of requests that are coming to a server in one second, and $\theta_{3}(t)$, which is the fraction of requests that are delayed per second. It is possible to assume that the marginal metric utility function for the average response time $u_{1}\left(\theta_{1}(t)\right)$ in cost dimension is constant:

$$
u_{1}\left(\theta_{1}(t) \mid \phi\right)=f_{5}\left(\theta_{1}(t) \mid \phi_{1,1}=1\right)=1 .
$$

As it was discussed for the performance utility dimension, the current number of users $\theta_{2}$ gives the execution context for the application owner and for the optimization platform. Every number of users gives the same utility for the application owner or DevOps engineer if they are properly served. Therefore, the value can also be modelled as constant function $f_{5}$ :

$$
u_{2}\left(\theta_{2}(t) \mid \boldsymbol{\phi}\right)=f_{5}\left(\theta_{2}(t) \mid \phi_{2,1}=1\right)=1 .
$$

The evaluation of the fraction of delayed requests $\theta_{3}(t)$ should reflect the fact that there is some fraction $R$, which is expected, while any value above $R^{+}$is not desired, because the profit from serving users is significantly lower. The fraction of the delayed requests should be as low as possible, so the 'template' function $f_{2}$ is suitable to represent this goal:

$$
u_{3}\left(\theta_{3}(t) \mid \boldsymbol{\phi}\right)=f_{2}\left(\theta_{3}(t) \mid \phi_{3,1}=R, \phi_{3,2}=R^{+}\right)
$$

We define the cost utility can be defined from (1), using (12):

$$
\begin{aligned}
U_{\text {price }} & \left(\boldsymbol{c}\left(t_{k}\right) \mid \boldsymbol{\theta}\left(t_{k+h}\right), \boldsymbol{\phi}\right) \\
= & u_{3}\left(\theta_{3}\left(t_{k}\right) \mid \boldsymbol{\phi}\right)+\frac{\partial u_{3}}{\partial \theta_{3}}\left(\theta_{3}\left(t_{k}\right)\right)\left[\theta_{3}\left(t_{k+h}\right)-\theta_{3}\left(t_{k}\right)\right]
\end{aligned}
$$

5.3.3 Overall utility. The overall utility contain two dimensions: performance utility and cost utility. We propose a way to combine these points using affine weighted combination of both values. Using (11) and (13), the utility value can be calculated:

$$
\begin{gathered}
U\left(\boldsymbol{c}\left(t_{k}\right) \mid \boldsymbol{\theta}\left(t_{k+h}\right), \boldsymbol{\phi}\right)=\zeta U_{\text {time }}\left(\boldsymbol{c}\left(t_{k}\right) \mid \boldsymbol{\theta}\left(t_{k+h}\right), \boldsymbol{\phi}\right) \\
+(1-\zeta) U_{\text {price }}\left(\boldsymbol{c}\left(t_{k}\right) \mid \boldsymbol{\theta}\left(t_{k+h}\right), \boldsymbol{\phi}\right)
\end{gathered}
$$

\section{EXAMPLE EVALUATION}

\subsection{Queueing framework}

Since the user requests enter a First-In-First-Out (FIFO) queue it is natural to represent the application as an open queuing system with one or several servers. The number of requests will certainly vary over the day, but for the sake of exposition here it will be understood that the daily variation can be split into periods with an approximately constant average level of requests per second, $\bar{\theta}_{2}$. Without further knowledge of a real application, one may model the number of requests per second as Poisson distributed with the given average, $\theta_{2}\left(t_{k+h}\right) \sim \operatorname{Poisson}\left(\bar{\theta}_{2}\right)$. Interpreting this as a counting process for the request arrivals, the time between two requests will be a random time exponentially distributed with the expected number of requests per seconds as parameter, $t_{k+1}-t_{k} \sim \operatorname{Exp}\left(\bar{\theta}_{2}\right)$. Similarly, it is reasonable to expect that the average time to serve one request measured in seconds can be estimated as $\bar{T}_{S}$, and that the time taken to serve one request is again exponentially distributed with the server capacity measured in requests per second, $\operatorname{Exp}\left(1 / \bar{T}_{S}\right)$.

With the above considerations the application can be modelled as an $M / M / s$ queue where $s$ is the number of servers, allowing the reuse of some standard results for this queue model [20, pp. 5159]. One may assume that the average requests per second, $\bar{\theta}_{2}$, will stay constant long enough for the queue to be considered to be in a stationary state.

The expected utilization of each server is obviously the expected arrivals divided by the expected server handling taking into account that there are $s$ servers available to handle the requests,

$$
\rho=\frac{\bar{\theta}_{2}}{s / \bar{T}_{S}}=\frac{\bar{\theta}_{2} \bar{T}_{S}}{s}<1
$$

where the upper limit is necessary to ensure that the servers eventually are able to catch up with the requests, i.e. the queue length will be finite. This implies that $s>\left\lceil\bar{\theta}_{2} \bar{T}_{S}\right\rceil$, which can be used to find the minimum number of servers needed. Furthermore, the average response time to the user is the expected waiting time in the queue plus the expected service time,

$$
\bar{\theta}_{1}=\frac{(s \rho)^{s} p_{0}}{s ! s\left(1 / \bar{T}_{S}\right)(1-\rho)^{2}}+\bar{T}_{S}
$$

where the probability of an empty queue is given by

$$
p_{0}=\left[\frac{(s \rho)^{s}}{s !(1-\rho)}+\sum_{n=0}^{s-1} \frac{(s \rho)^{n}}{n !}\right]^{-1}
$$

A request that is not serviced by time threshold, $T^{+}$will eventually be flagged as delayed. The time budget for a decoding can be split into two parts, $T^{+}=T_{Q}^{+}+T_{S}^{+}$where $T_{Q}^{+}$is the upper time limit allowed for queueing and $T_{S}^{+}$is the maximum time allowed for the server decoding the document. The probability that the request is queued longer than $T_{Q}^{+}$is given by

$$
\begin{aligned}
\operatorname{Pr}\left[T_{Q}>T_{Q}^{+}\right] & =1-\operatorname{Pr}\left[T_{Q} \leq T_{Q}^{+}\right] \\
& =\frac{(s \rho)^{s} p_{0}}{s !(1-\rho)} e^{-s(1-\rho) T_{Q}^{+} / \bar{T}_{S}}
\end{aligned}
$$

and the server delay probability is given by the cumulative density function for the exponential distribution

$$
\operatorname{Pr}\left[T_{S}>T_{S}^{+}\right]=1-\operatorname{Pr}\left[T_{S} \leq T_{S}^{+}\right]=e^{-T_{S}^{+} / \bar{T}_{S}}
$$

Since the service time is independent of how long the request has been queued, the probability of exceeding the delay threshold is the sum of (18) and (19). The fraction of delayed requests, $\theta_{3}(t)$ equals this joint probability.

\subsection{Numerical parameters}

A server is a single core that serves requests sequentially. When a server performs well, the average response time is $\bar{T}_{S}=89 \mathrm{~ms}=$ $89 \cdot 10^{-3} \mathrm{~s}$, which means that a single server saturates when the average number of requests per second is $\bar{\theta}_{2}=10^{3} / 89=11.23$. According to the common knowledge ${ }^{2}$, the expected response time to the user should be around $\bar{T}=0.1 \mathrm{~s}$, while after one second a request should be considered delayed. Since the document decoding time is independent of the number of servers, the threshold for delayed requests is fully allocated to the queueing delay, $T_{Q}^{+}=1 \mathrm{~s}$ The acceptable percentage of delayed requests can be assumed to be

\footnotetext{
${ }^{2} \mathrm{https}: / /$ www.dnsstuff.com/response-time-monitoring
} 
Table 1: Performance metrics of the queueing model of the secure storage example and corresponding utility values

\begin{tabular}{|c|c|c|r|r|r|r|r|}
\hline Time & $\begin{array}{c}\text { Load } \\
\left(\bar{\theta}_{2}(t)\right)\end{array}$ & $\begin{array}{c}\text { Servers } \\
\left(c_{1}^{*}\right)\end{array}$ & Utilization $(\rho)$ & $\begin{array}{c}\text { Average response } \\
\left(\theta_{1}(t)\right)\end{array}$ & $\begin{array}{c}\text { Delayed requests } \\
\left(\theta_{3}(t)\right)\end{array}$ & $\begin{array}{c}\text { Marginal metric } \\
\text { utility } U\end{array}$ & Direct utility $v$ \\
\hline$t_{1}$ & 100 & 12 & 0.741667 & 0.0962045 & $1.87 \cdot 10^{-16}$ & 0.993883 & 0.995671 \\
$t_{2}$ & 110 & 12 & 0.815833 & 0.105346 & $6.67 \cdot 10^{-12}$ & 0.990239 & 0.998170 \\
$t_{3}$ & 120 & 12 & 0.890000 & 0.130056 & $2.20 \cdot 10^{-7}$ & 0.980395 & 0.994967 \\
$t_{4}$ & 130 & 12 & 0.964167 & 0.267191 & $6.87 \cdot 10^{-3}$ & 0.925552 & 0.856933 \\
$t_{5}$ & 131 & 12 & 0.971583 & 0.320972 & 0.0192672 & 0.903744 & 0.788159 \\
$t_{6}$ & 132 & 12 & 0.979000 & 0.412911 & 0.0540442 & 0.866044 & 0.682151 \\
$t_{7}$ & 133 & 12 & 0.986417 & 0.605511 & 0.151525 & 0.786307 & 0.543874 \\
$t_{8}$ & 134 & 12 & 0.993833 & 1.26196 & 0.424647 & 0.516360 & 0.511127 \\
\hline
\end{tabular}

$1 \%$, which is still acceptable for the user, while anything more than $70 \%$ is definitely not desired, so $R=0.01, R^{+}=0.7$.

Consider the situation at $t_{1}$ with moderate load, $\bar{\theta}_{2}=100$ requests per second. The minimum number of servers from (15) is then 9 . However, the average response time of (16) at $0.945 \mathrm{~s}$ will then be almost 10 times the response time requirement. The optimal number of servers can then be found from setting $\bar{T}=\theta_{1}\left(t_{1}\right)=0.1 \mathrm{~s}$, and solve (16) for the number of servers, showing that this requirement can be met with 12 servers. i.e. cores in this setting. Without lack of generality we can associate the full scalability with the number of instances, and so $c_{1}^{*}=12$ and $c_{2}^{*}=1$. The performance metric values with increasing load is given in Table 1.

\subsection{Utility change calculations}

The change in the utility can be expressed locally around the point consisting of the optimal solution $c^{*}$ found for the set of measurements $\boldsymbol{\theta}\left(t_{k}\right)$ in terms of the measurements at a later monitoring event, $t_{k+h}, \boldsymbol{\theta}\left(t_{k+h}\right)=\left[\theta_{1}\left(t_{k+h}\right), \theta_{2}\left(t_{k+h}\right), \theta_{3}\left(t_{k+h}\right)\right]^{T}$ according to the previous theory using (1). We calculate the change in the value of utility for the example modelled in Section 5 using numerical values presented in Section 6.2. For the simplification of calculations, we assume that all weights for both dimensions are equal, $\zeta=0.5$. Results are presented in Table 1.

6.3.1 Marginal metric utility. The utility value $U_{0}=U\left(c^{*}\left(t_{1}\right)\right)$ is needed to calculate the change in the utility in marginal metric utility calculations. We consider the worst case which is when DevOps engineer did not assign this value and it is calculated using (2), where weights are set to be equal. We make calculations for both dimensions separately. We calculate the utility value of optimal configuration in terms of performance utility for $\boldsymbol{\theta}\left(t_{1}\right)$ showed in Table 1 using (10). We set $\epsilon=10^{-20}, \bar{T}_{S}=0.089$, and not acceptable time when the request is considered delayed $T^{+}=1$, so $\phi=\left[\phi_{1,1}, \phi_{1,2}, \phi_{2,1}\right]^{T}=\left[\bar{T}_{S}, T^{+}, 1\right]^{T}$ which gives

$U_{\text {time }}\left(c^{*}\left(t_{1}\right) \mid \boldsymbol{\theta}\left(t_{1}\right), \boldsymbol{\phi}\right)=0.9971276 \quad \frac{\partial u_{1}}{\partial \theta_{1}}\left(\theta_{1}\left(t_{1}\right)\right)=-0.79675$

We can calculate the current performance utility for $\left(c^{*}\left(t_{1}\right)\right)$ and measurements $\boldsymbol{\theta}\left(t_{2}\right)$ using (11) and numerical values from Table 1:

$$
\begin{aligned}
& U_{\text {time }}\left(c^{*}\left(t_{1}\right) \mid \theta\left(t_{2}\right)\right)=0.9971276-0.79675 \\
& \times(0.105346-0.0962)=0.9898405245
\end{aligned}
$$

We calculate the initial cost utility for $\boldsymbol{c}^{*}\left(t_{1}\right)$ and $\boldsymbol{\theta}\left(t_{1}\right)$ using (2) and (12). We set $\boldsymbol{\phi}=\left[\phi_{1,1}, \phi_{2,2}, \phi_{3,1}, \phi_{3,2}\right]^{T}=\left[1,1, R, R^{+}\right]^{T}, \epsilon=$ $10^{-2}, R=0.01, R^{+}=0.7$, and get:

$U_{\text {price }}\left(c^{*}\left(t_{1}\right) \mid \boldsymbol{\theta}\left(t_{1}\right)\right)=0.9906382 \quad \frac{\partial u_{3}}{\partial \theta_{3}}\left(\theta_{3}\left(t_{1}\right)\right)=-0.0617619$

The change in the utility for the optimal configuration $c^{*}\left(t_{1}\right)$ for time $t_{2}$ is calculated using (13) and numerical values:

$$
\begin{gathered}
U_{\text {price }}\left(\boldsymbol{c}^{*}\left(t_{1}\right) \mid \boldsymbol{\theta}\left(t_{2}\right), \boldsymbol{\phi}\right)=0.990638-0.0617619 \\
\times\left(6.67 \cdot 10^{-12}-1.87 \cdot 10^{-16}\right)=0.990638
\end{gathered}
$$

In the same way we calculate the change in the utility in both dimensions for the vector $\boldsymbol{\theta}\left(t_{3}\right), \ldots, \boldsymbol{\theta}\left(t_{8}\right)$. Overall utility values calculated using (14) are reported in Table 1.

6.3.2 Directly modeled utility. We compare our approach with the directly modelled utility function (9). We calculate utility function values separately for the performance and for the cost utility dimensions. Overall utility values for $\boldsymbol{\theta}\left(t_{3}\right), \ldots, \boldsymbol{\theta}\left(t_{8}\right)$ calculated using (9) are presented in Table 1.

To calculate the utility in the performance dimension, there is a need to set parameters $\phi_{1}, \phi_{2}$ of the beta distribution function. The highest possible utility value should be achieved for the expected response time $\bar{T}=0.1 \mathrm{~s}$. We calculate $v_{\text {time }}\left(\boldsymbol{c}^{*}\left(t_{1}\right) \mid \boldsymbol{\theta}\left(t_{1}\right)\right)$ using formula (10) defined by Horn and Rozanska [7]. We set $a=1.5, b=5$ as it gives the desired shape, then $\beta^{+}(1.5,5)=2.81665$ : $v_{\text {time }}\left(c^{*}\left(t_{1}\right) \mid \boldsymbol{\theta}\left(t_{1}\right), \boldsymbol{\phi}\right)=0.994509$. For the cost dimension, we put parameters $\phi=\left[c_{1}^{-}, c_{1}{ }^{+}, P^{-}, P^{+}, P\left(c^{*}\left(t_{1}\right)\right)\right]^{T}=[1,15,0.5 \$, 10 \$, 4 \$$. $12]^{T}$. We assume that values of $\theta_{3}\left(t_{k+h}\right)$ that are below $10^{-10}$ are zero and we calculate the utility value using (8):

$$
\begin{aligned}
v_{\text {price }} & \left(\boldsymbol{c}^{*}\left(t_{1}\right) \mid \boldsymbol{\theta}\left(t_{1}\right), \boldsymbol{\phi}\right)=\left(\frac{100 \cdot 10 \cdot 15-4 \cdot 12}{100 \cdot 10 \cdot 15-0.5}\right) \\
= & 0.99683322777
\end{aligned}
$$

\section{DISCUSSION}

To summarize, numerical results clearly show that the approach presented in this paper gives promising results that are comparable with results derived from direct utility modelling. The modelled situation started at $t_{1}$ with highly evaluated utility values of metrics $\boldsymbol{\theta}\left(t_{1}\right)$, while for $t_{8}$ the measured metric values indicated that the utility of the application had significantly decreased and the drop in 
the utility of the optimal configuration for $t_{1}$ can be noticed in both approaches.

Even though the values of utility look similar for both approaches, it is important to notice that it is not necessary to calculate the difference between $U\left(\boldsymbol{c}^{*}\left(t_{1}\right) \mid \boldsymbol{\theta}\left(t_{k+h}\right), \boldsymbol{\phi}\right)$ and $v\left(\boldsymbol{c}^{*}\left(t_{1}\right) \mid \boldsymbol{\theta}\left(t_{k+h}\right), \boldsymbol{\phi}\right)$ The direction of the change in the utility should be evaluated and should correctly indicate the need of an adaptation of the application configuration to a new configuration that is optimal for the current execution context. The proposed metric based utility is as good as the more complex direct utility modelling for this purpose.

The utility value for the changed context $\boldsymbol{\theta}\left(t_{k+h}\right)$ stay in the standard unit interval for the presented example. However, this property is not assured in general by our modelling approach. By definition, the linearisation method works in the neighbourhood of $c^{*}\left(t_{1}\right)$ and $\boldsymbol{\theta}\left(t_{i}\right)$. A change in the utility that makes the new utility out of the interval $[0,1]$ is a strong indication that a new optimal configuration must be computed because the current configuration is no longer optimal. This is a feature that is not clearly defined when using direct utility function modelling, and an out-of-bound utility value can be used as a trigger for optimization in the MAPE-K loop. The evaluation presented for this example is based on a theoretical basis from queuing theory for reproducability, although some assumptions about the arrival and service time distributions may not be realistic for a real application use. The purpose here was to demonstrate the methodology through a complete modelling example to validate that the approach is useful and that the utility impressions of the DevOps engineer regarding the set of monitoring measurements obtained from the application can be used to construct workable utility functions that will be useful in an autonomic computing closed loop control setting.

\section{FUTURE WORK}

We showed that the proposed approach can be an alternative way of modelling user preferences that are possibly more friendly for business users or DevOps engineers that are less technical and less mathematically advanced. We work on an evaluation with DevOps engineers about how more friendly our approach is. What is more, in this paper, a standard affine combination was used to combine the utility dimensions individually modelled by the metric based utility functions. The next step will be to investigate the use of Archimedean utility copulas as a way to better represent the overall system utility.

\section{CONCLUSION}

Our hypothesis was that one may overcome the difficulties in utility function modelling for autonomic Cloud application management by deriving the deployment configuration utility from the marginal univariate utility functions decided individually over each of the measured metric values reflecting the application's current execution context. We showed that this approach can give the same or even more knowledge regarding the evolution of the application's utility. The proposed modelling methodology is therefore a promising bridge to the established utility copula theory. We have demonstrated that the proposed approach in its present form can readily be used in Cloud optimization to detect the change in the quality of the currently deployed configuration. Thus, the biggest advantage of this modelling is the fact that it can be extended and used as a part of MAPE-K loop in the optimization process.

\section{ACKNOWLEDGMENTS}

This work has received funding from the European Union's Horizon 2020 research and innovation programme under grant agreement No 871643 MORPHEMIC Modelling and Orchestrating heterogeneous Resources and Polymorphic applications for Holistic Execution and adaptation of Models In the Cloud, see http://morphemic.cloud

\section{REFERENCES}

[1] Ali E. Abbas. 2009. Multiattribute Utility Copulas. Operations Research 57, 6 (Aug. 2009), 1367-1383. https://doi.org/10.1287/opre.1080.0687

[2] Ali E. Abbas and Ronald A. Howard. 2005. Attribute Dominance Utility. Decision Analysis 2, 4 (Dec. 2005), 185-206. https://doi.org/10.1287/deca.1050.0046

[3] Ali E. Abbas and Zhengwei Sun. 2015. Multiattribute Utility Functions Satisfying Mutual Preferential Independence. Operations Research 63, 2 (March 2015), 378-393. https://doi.org/10.1287/opre.2015.1350

[4] Ali E. Abbas and Zhengwei Sun. 2019. Archimedean Utility Copulas with Polynomial Generating Functions. Decision Analysis 16, 3 (Aug. 2019), 218-237. https://doi.org/10.1287/deca.2018.0386

[5] Ching-Ter Chang. 2011. Multi-choice goal programming with utility functions. European Journal of Operational Research 215, 2 (Dec. 2011), 439-445. https: //doi.org/10.1016/j.ejor.2011.06.041

[6] Christian Genest and R. Jock Mackay. 1986. Copules archimédiennes et families de lois bidimensionnelles dont les marges sont données. Canadian Journal of Statistics 14, 2 (1986), 145-159. https://doi.org/10.2307/3314660

[7] Geir Horn and Marta Rózańska. 2019. Affine Scalarization of Two-Dimensional Utility Using the Pareto Front. In Proceedings of the IEEE International Conference on Autonomic Computing (ICAC 2019). IEEE, Conference location: Umeå, Sweden, 147-156. https://doi.org/10.1109/ICAC.2019.00026

[8] Geir Horn and Paweł Skrzypek. 2018. MELODIC: Utility Based Cross Cloud Deployment Optimisation. In Proceedings of the 32nd International Conference on Advanced Information Networking and Applications Workshops (WAINA). IEEE Computer Society, Conference Location: Krakow, Poland, 360-367. https: //doi.org/10.1109/WAINA.2018.00112

[9] IBM. 2005. An Architectural Blueprint for Autonomic Computing. White Paper Third Edition. IBM, 17 Skyline Drive, Hawthorne, NY 10532, U.S.A. 34 pages.

[10] Itzhak Gilboa. 2010. Rational Choice. MIT Press, Cambridge, MA, USA.

[11] Jacqueline Floch, Cristina. Frà, Rolf Fricke, Kurt Geihs, Michael Wagner, Jorge Lorenzo, E. Soladana, Stephane Mehlhase, Nearchos Paspallis, Hossein Rahnama, Pedro A. Ruiz, and Ulrich Scholz. 2013. Playing MUSIC - Building ContextAware and Self-Adaptive Mobile Applications. Software: Practice and Experience 43, 3 (March 2013), 359-388. https://doi.org/10.1002/spe.2116

[12] Jeffrey O. Kephart and David M. Chess. 2003. The Vision of Autonomic Computing. Computer 36, 1 (2003), 41-50. https://doi.org/10.1109/MC.2003.1160055

[13] Jeffrey O. Kephart and Rajarshi Das. 2007. Achieving Self-Management via Utility Functions. IEEE Internet Computing 11, 1 (Jan. 2007), 40-48. https: //doi.org/10.1109/MIC.2007.2

[14] Kurt Geihs, Paolo Barone, Frank Eliassen, Jacqueline Floch, Rolf Fricke, Eli Gjørven, Svein Hallsteinsen, Geir Horn, Mohammad Ullah Khan, Alessandro Mamelli, George A. Papadopoulos, Nearchos Paspallis, Roland Reichle, and Erlend Stav. 2009. A Comprehensive Solution for Application-Level Adaptation. Software: Practice and Experience 39, 4 (2009), 385-422. https://doi.org/10. 1002/spe.900

[15] Marco LiCalzi and Annamaria Sorato. 2006. The Pearson system of utility functions. European Journal of Operational Research 172, 2 (July 2006), 560573. https://doi.org/10.1016/j.ejor.2004.10.012

[16] Patrick L. Brockett and Linda L. Golden. 1987. A Class of Utility Functions Containing All the Common Utility Functions. Management Science 33, 8 (Aug. 1987), 955-964. https://doi.org/10.1287/mnsc.33.8.955

[17] Ralph L. Keeney. 1974. Multiplicative Utility Functions. Operations Research 22, 1 (Feb. 1974), 22-34. https://doi.org/10.1287/opre.22.1.22

[18] Roger B. Nelsen. 2006. An Introduction to Copulas (second ed.). Springer-Verlag, New York. https://doi.org/10.1007/0-387-28678-0

[19] Svein Hallsteinsen, Kurt Geihs, Nearchos Paspallis, Frank Eliassen, Geir Horn, Jorge Lorenzo, Alessandro Mamelli, and George A. Papadopoulos. 2012. A Development Framework and Methodology for Self-Adapting Applications in Ubiquitous Computing Environments. Journal of Systems and Software 85, 12 (Dec. 2012), 2840-2859. https://doi.org/10.1016/j.jss.2012.07.052

[20] U. Narayan Bhat. 2015. An Introduction to Queueing Theory: Modeling and Analysis in Applications (second ed.). Birkhäuser Basel. https://doi.org/10.1007/ 978-0-8176-8421-1 